\title{
La composition du lait de brebis de la région de Plovdiv en Bulgarie et de Ioannina en Grèce
}

\author{
par \\ M. BALTADJIEVA*, B. VEINOGLOU**, J. KANDARAKIS***, \\ M. EDGARYAN ${ }^{* * * *}$ et V. STAMENOVA****
}

\section{INTRODUCTION}

Cette étude a été réalisée dans le cadre de la coopération du Laboratoire de Technologie Laitière de l'Institut de Technologie Alimentaire de Plovdiv et du Laboratoire homologue de l'Ecole Supérieure d'Agriculture d'Athènes.

Elle concerne la composition du lait de brebis produit dans la région de Plovdiv en Bulgarie et dans celle de Ioannina en Grèce, d'où les deux laboratoires se sont procurés les quantités nécessaires à la préparation de fromages expérimentaux. Les régions mentionnées sont représentatives de l'élevage ovin des deux pays avec une production laitière très importante.

Nous signalons que les deux pays ont une production de lait de brebis très remarquable, $550000 \mathrm{t}$ en Grèce et $300000 \mathrm{t}$ environ en Bulgarie.

A l'époque de la grande production (mois de mai), une usine coopérative, dans la région de Ioannina, collecte $120 \mathrm{t}$ de lait de brebis par jour. Le ramassage se fait en citernes réfrigérées et de ce lait nous avons pris des échantillons représentatifs. Les échantillons de la région de Plovdiv ont été prélevés de la même manière.

* Vice-Recteur de l'Institut de Technologie Alimentaire de Plovdiv, Bulgarie.

** Professeur à la chaire de Technologie laitière à l'Ecole Supérieure d'Agriculture d'Athènes, Grèce.

*** Assistant à la chaire de Technologie laitière à l'Ecole Supérieure d'Agriculture d'Athènes, Grèce.

***** Assistantes à la chaire de Technologie laitière de l'Institut de Technologie Alimentaire de Plovdiv, Bulgarie. 
Les races ovines qui dominent dans la région de Ioannina sont surtout la Vlahiki, un petit nombre de Karagouniki et les produits de leurs croisements. La durée de la lactation est de 6 à 8 mois et la quantité de lait d'environ $100 \mathrm{~kg}$ par tête.

\section{MATERIELS ET METHODES}

\section{Origine des échantillons}

Durant la lactation des années 1979 et 1980, 63 échantillons de lait grec et 48 de lait bulgare ont été prélevés tous les $10 \mathrm{j}$.

Les analyses chimiques ont été effectuées dans les deux laboratoires.

\section{Méthodes analytiques}

Les analyses des protéines totales et de la caséine ont été réalisées selon les normes 20/1962 et 29/1964 de la Fédération Internationale de Laiterie, le lactose et la matière sèche selon les normes 28A/1974 et 21/1962. L'analyse de la teneur en cendres et en calcium a été effectuée selon le British Standard 1741/1963.

La matière grasse a été déterminée selon la méthode Gerber, l'acidité avec la méthode Dornic et le poids spécifique à $15^{\circ} \mathrm{C}$ avec le lactodensimètre.

\section{RESULTATS ET DISCUSSION}

\section{Composition moyenne du lait de brebis}

La composition moyenne des laits de brebis des deux régions, d'après les analyses que nous avons effectuées durant les lactations des années 1979 et 1980 , est donnée au tableau 1.

L'étude statistique des données de nos analyses nous permet de constater qu'il existe une différence significative, au seuil de 0,05 , dans la composition des laits des deux régions surtout en ce qui concerne la matière grasse, l'extrait sec, l'extrait sec dégraissé et les cendres.

Au contraire, il n'existe pas de grandes différences pour l'acidité, le $\mathrm{pH}$ et la densité.

Le lait de la région de Plovdiv est plus riche pour tous les constituants, sauf les protéines solubles et les cendres.

En comparant nos résultats aux données que l'on peut trouver dans la bibliographie, tableau 2, nous pouvons constater que le lait de Ioannina est d'une composition moyenne, avec une teneur en 
TABLEAU 1

Composition moyenne du lait de brebis en Bulgarie (B) et en Grèce (G)

\begin{tabular}{|c|c|c|c|c|c|c|c|c|c|c|c|}
\hline & \multicolumn{5}{|c|}{ Grèce (Ioannina) } & \multicolumn{6}{|c|}{ Bulgarie (Plovdiv) } \\
\hline & Max & Min & Moyen & $\mathrm{S}$ & V & $\operatorname{Max}$ & Min & Moyen & $\mathrm{S}$ & $\mathrm{V}$ & t.test \\
\hline Matière grasse p. 100 & 6,45 & - 7,40 & 6,88 & 0,26 & 3,73 & 6,40 & $-11,40$ & 8,10 & 1,44 & 17,77 & 3,84 \\
\hline Protéines totales p. 100 & 5,14 & - 6,13 & 5,74 & 0,27 & 4,78 & 5,14 & - 7,64 & 5,83 & 0,57 & 9,80 & 0,60 \\
\hline Protéines solubles p. 100 & 0,85 & $-1,59$ & 1,44 & 0,16 & 10,79 & 0,86 & - 1,88 & 1,33 & 0,29 & 21,79 & 1,50 \\
\hline Caséine p. 100 & 3,78 & - 4,67 & 4,32 & 0,22 & 5,10 & 3,86 & - 6,49 & 4,51 & 0,65 & 14,51 & 1,20 \\
\hline Lactose p. 100 & 4,16 & - 4,88 & 4,59 & 0,20 & 4,37 & 4,37 & - 5,02 & 4,72 & 0,18 & 3,80 & 1,90 \\
\hline Cendre p. 100 & 0,88 & $-1,05$ & 0,95 & 0,04 & 4,27 & 0,64 & - 1,02 & 0,87 & 0,10 & 11,64 & $3,15^{*}$ \\
\hline Extrait sec p. 100 & 16,97 & $-18,61$ & 17,80 & 0,48 & 2,71 & 17,48 & $-23,84$ & 19,54 & 1,66 & 8,49 & $4,63^{*}$ \\
\hline Extrait sec dégraissé p. 100 & 9,66 & $-11,75$ & 10,92 & 0,49 & 4,48 & 10,44 & $-13,44$ & 11,43 & 0,66 & 5,78 & 2,69 * \\
\hline Calcium p. 100 & 0,133 & $-0,239$ & 0,186 & 0,024 & 13,11 & 0,145 & $-0,232$ & 0,194 & 0,022 & 11,31 & 1,22 \\
\hline $\mathrm{pH}$ & 6,48 & - 6,61 & 6,58 & 0,03 & 0,43 & 6,53 & $-6,63$ & 6,57 & 0,03 & 0,45 & 0,97 \\
\hline Acidité & 0,19 & - 0,24 & 0,22 & 0,01 & 4,93 & 0,18 & - 0,24 & 0,21 & 0,02 & 7,55 & 1,28 \\
\hline Densité & 1,033 & - 1,038 & 1,036 & 0,001 & 0,09 & 1,032 & - 1,039 & 1,036 & 0,002 & 0,19 & 0,17 \\
\hline $\mathrm{n}_{\mathrm{B}}=48$ & art-ty & ype & Coef & $\mathrm{de}$ & on & $\mathrm{t}^{*}=\mathrm{D}$ & Différe & signi & ive. & & \\
\hline
\end{tabular}


TABLEAU 2

Composition de lait de brebis de différents pays

\begin{tabular}{|c|c|c|c|c|c|c|c|c|}
\hline & & $\begin{array}{l}\text { Extrait sec } \\
\text { p. } 100\end{array}$ & $\begin{array}{l}\text { Matière grasse } \\
\text { p. } 100\end{array}$ & $\begin{array}{l}\text { Protéines } \\
\text { p. } 100\end{array}$ & Caséine & $\begin{array}{c}\text { Protéines } \\
\text { solubles p. } 100\end{array}$ & $\begin{array}{c}\text { Lactose } \\
\text { p. } 100\end{array}$ & $\begin{array}{l}\text { Cendre } \\
\text { p. } 100\end{array}$ \\
\hline \multirow[t]{3}{*}{ Bulgarie } & {$[1]$} & 18,10 & 7,65 & - & 3,55 & - & - & - \\
\hline & [2] & 17,87 & 6,01 & 5,487 & 4,261 & - & 4,456 & 0,883 \\
\hline & [3] & - & 5,29 & 5,62 & - & - & 4,55 & - \\
\hline Hongrie & {$[4]$} & - & 7,27 & 6,215 & 4,972 & - & 4,80 & 0,890 \\
\hline \multirow[t]{2}{*}{ Tchécoslovaquie } & [5] & 19,36 & 8,28 & 5,93 & - & - & 4,36 & 0,876 \\
\hline & {$[6]$} & 19,20 & 7,77 & 5,47 & - & 0,89 & 4,74 & 0,933 \\
\hline \multirow[t]{3}{*}{ Grèce } & [7] & 19,080 & 7,85 & 5,472 & 4,41 & 0,817 & 4,799 & 0,924 \\
\hline & {$[8]$} & 20,665 & 9,034 & 6,521 & 4,987 & - & 4,728 & 0,953 \\
\hline & [9] & 17,591 & 6,404 & 5,714 & 4,398 & - & 5,095 & 0,874 \\
\hline
\end{tabular}


matière grasse inférieure à la moyenne, facteur qui influence aussi l'extrait sec total. Au contraire le lait de Plovdiv est un des plus riches mentionnés dans la bibliographie, surtout en ce qui concerne la matière grasse et l'extrait sec.

\section{Variation des différents constituants durant la lactation}

Dans la région de Ioannina, où nous avons une production de lait de brebis, du mois d'octobre jusqu'au mois d'août, il apparaît que les troupeaux ont un début de lactation différent. Ainsi le lait collecté provenait d'un mélange de troupeaux qui se trouvaient à un stade de lactation plus ou moins avancé, et les analyses que nous avons effectuées portent sur une période plus grande que la durée de la lactation.

En ce qui concerne le lait de Plovdiv, la production laitière s'étend du mois de janvier jusqu'au mois d'août et la remarque ci-dessus n'est pas valable.

En examinant les variations de prix des différents constituants, durant la période où nous avons collecté les échantillons, nous constatons :

En ce qui concerne la matière grasse, il existe une différence statistiquement significative entre le lait de deux régions (fig. 1). Les valeurs du lait de Ioannina sont inférieures, avec une variation limitée, autour d'une moyenne de 6,88 p. 100 pendant toute la période. Au contraire le lait de Plovdiv a une plus grande variation : les coefficients de variation sont respectivement 3,77 et 17,77 .

Pour le lait de la région de Ioannina, les valeurs les plus élevées se situent aux mois de novembre, décembre, février, juillet et août.

En ce qui concerne la matière grasse du lait de Plovdiv, les valeurs les plus élevées se situent aux mois de mai, juin, juillet et surtout pendant le mois d'août.

La teneur en protéines totales des deux régions est assez élevée (fig. 2). Pour le lait de Ioannina les variations sont limitées (coefficient de variation 4,78 ) avec les valeurs les plus basses se situant au début et à la fin de la lactation. Pour le lait de Plovdiv les variation sont plus grandes (coefficient de variation 9,80) et les valeurs les plus hautes se présentent vers la fin de la lactation. Pareille remarque peut être faite pour la caséine (fig. 3), pour le lait de Ioannina, tandis que pour le lait de Plovdiv les valeurs sont plus hautes vers la fin de la lactation.

Une variation importante a été constatée dans la teneur en protéines solubles, pour le lait des deux régions. Les coefficients de variation sont 10,79 pour le lait grec et 21,79 pour le lait bulgare. La valeur moyenne pour le lait de Ioannina est 1,44 p. 100 et pour le lait de Plovdiv 1,33, mais finalement les différences ne sont pas importantes. Les valeurs les plus élevées, pour les deux cas, se présentent au début et à la fin de la lactation. 


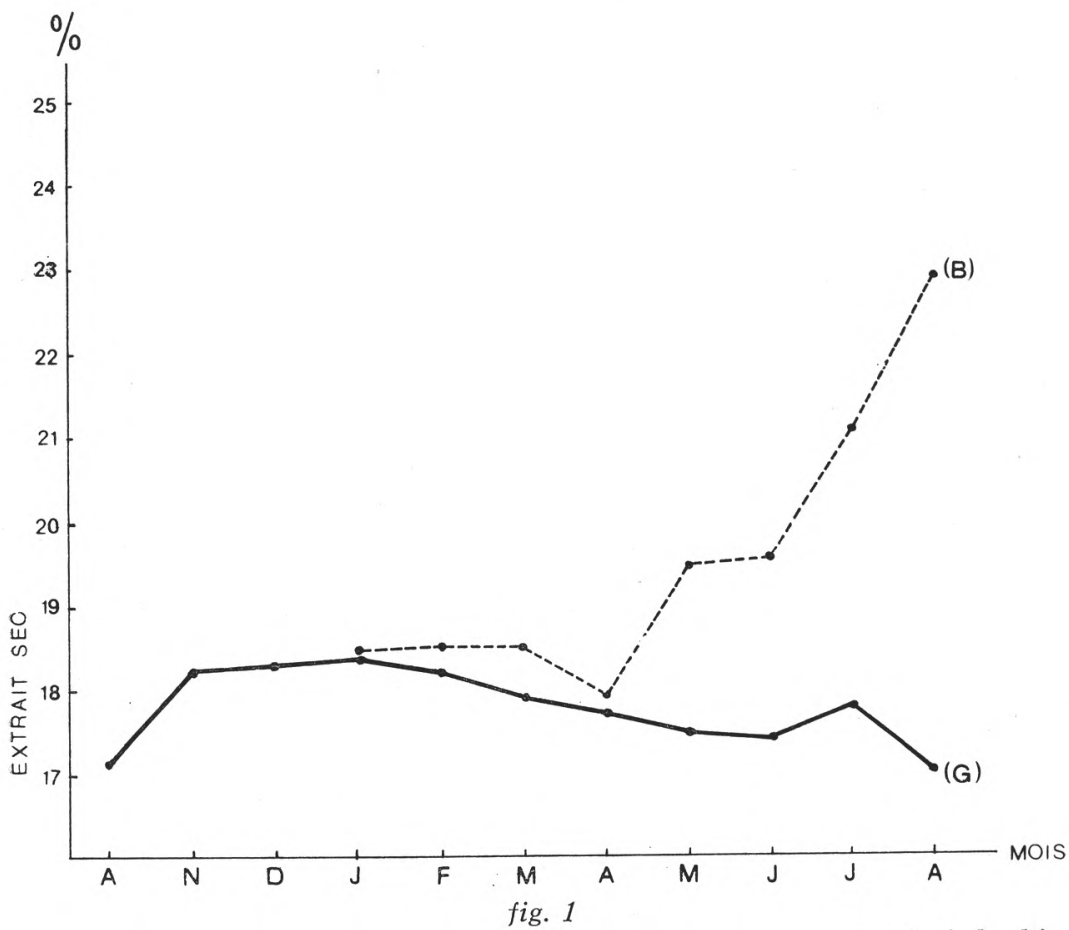

Variation, durant la lactation, de la teneur en matière grasse de lait de brebis de Plovdiv (B) et Ioannina (G).

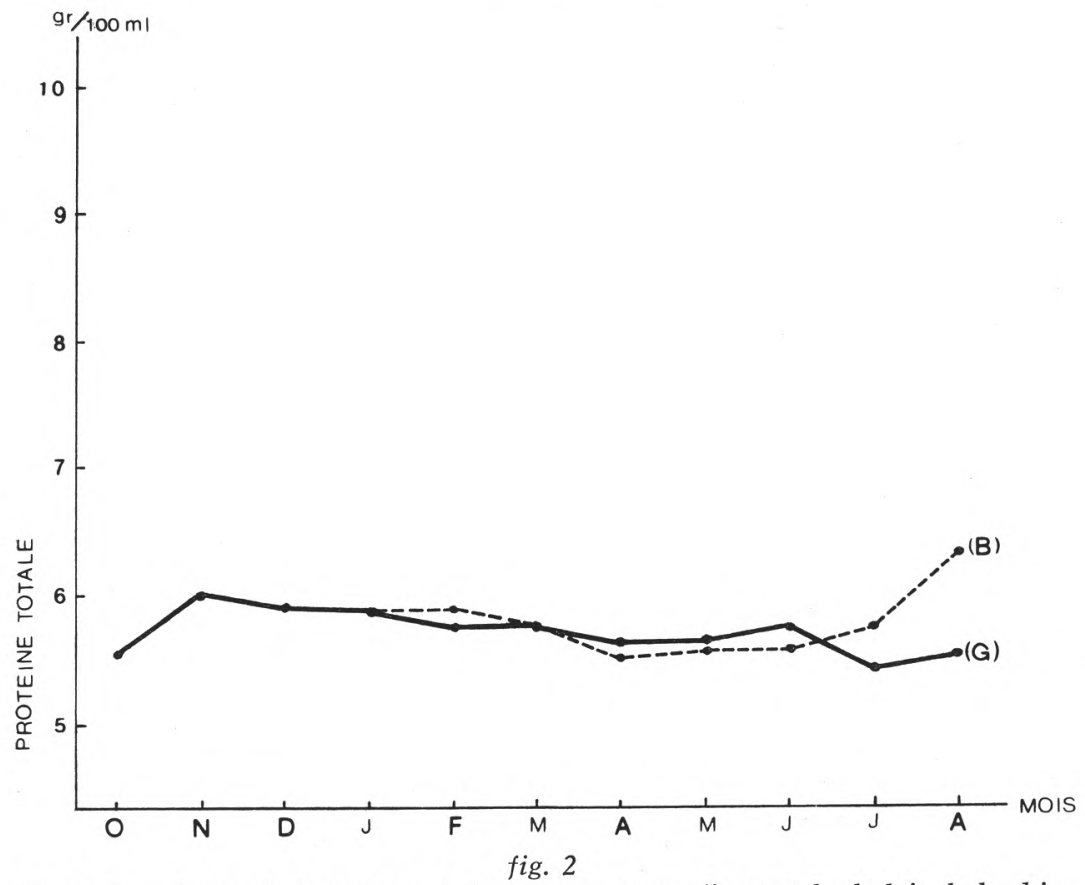

Variation, durant la lactation, de la teneur en protéine totale de lait de brebis de Plovdiv (B) et Ioannina (G). 


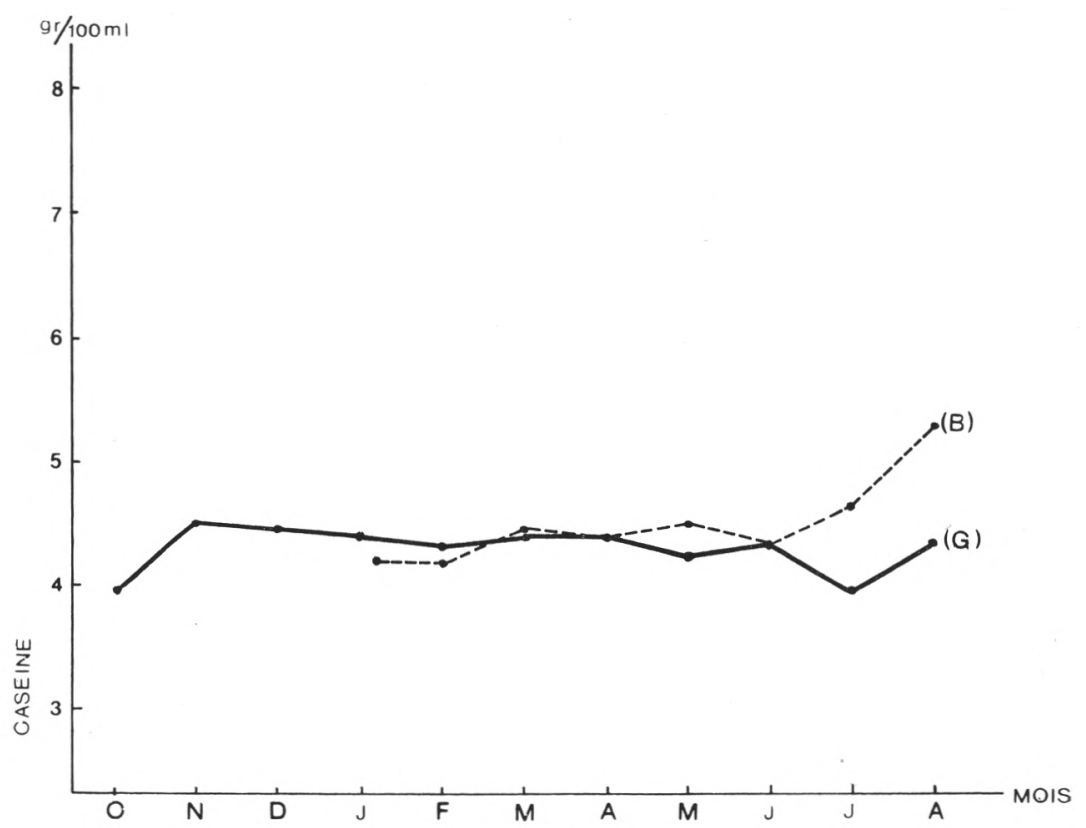

fig. 3

Variation, durant la lactation, de la teneur en caséine de lait de brebis $9 \mathrm{r} / 100 \mathrm{ml}$ de Plovdiv (B) et Ioannina (G).

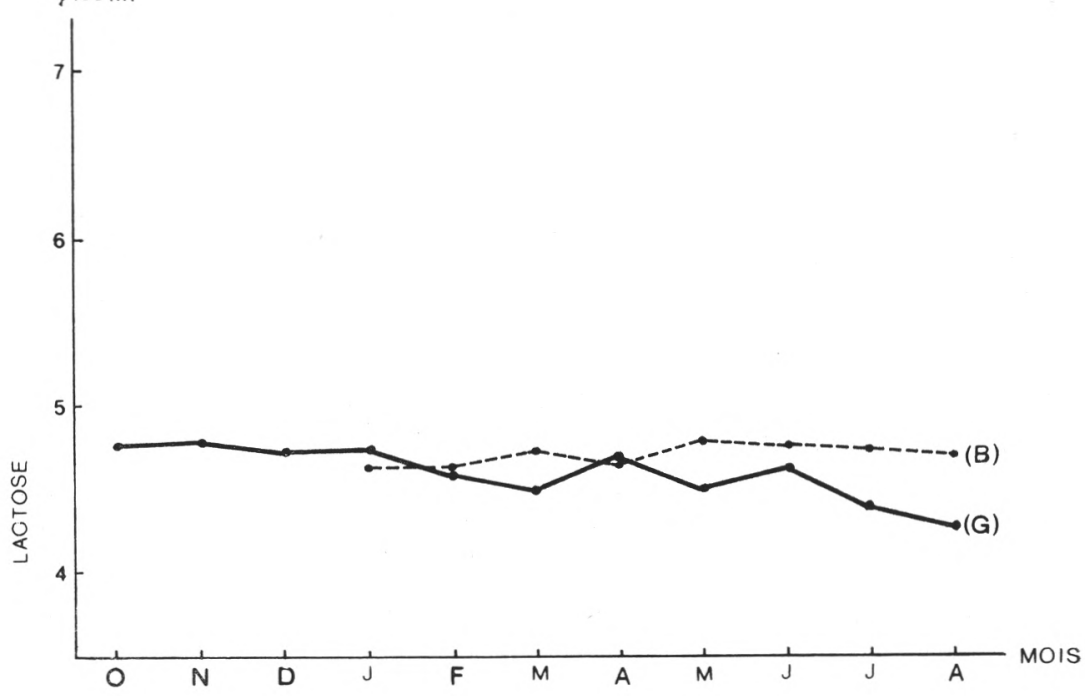

fig. 4

Variation, durant la lactation, de la teneur en lactose de lait de brebis de Plovdiv (B) et Ioannina (G). 


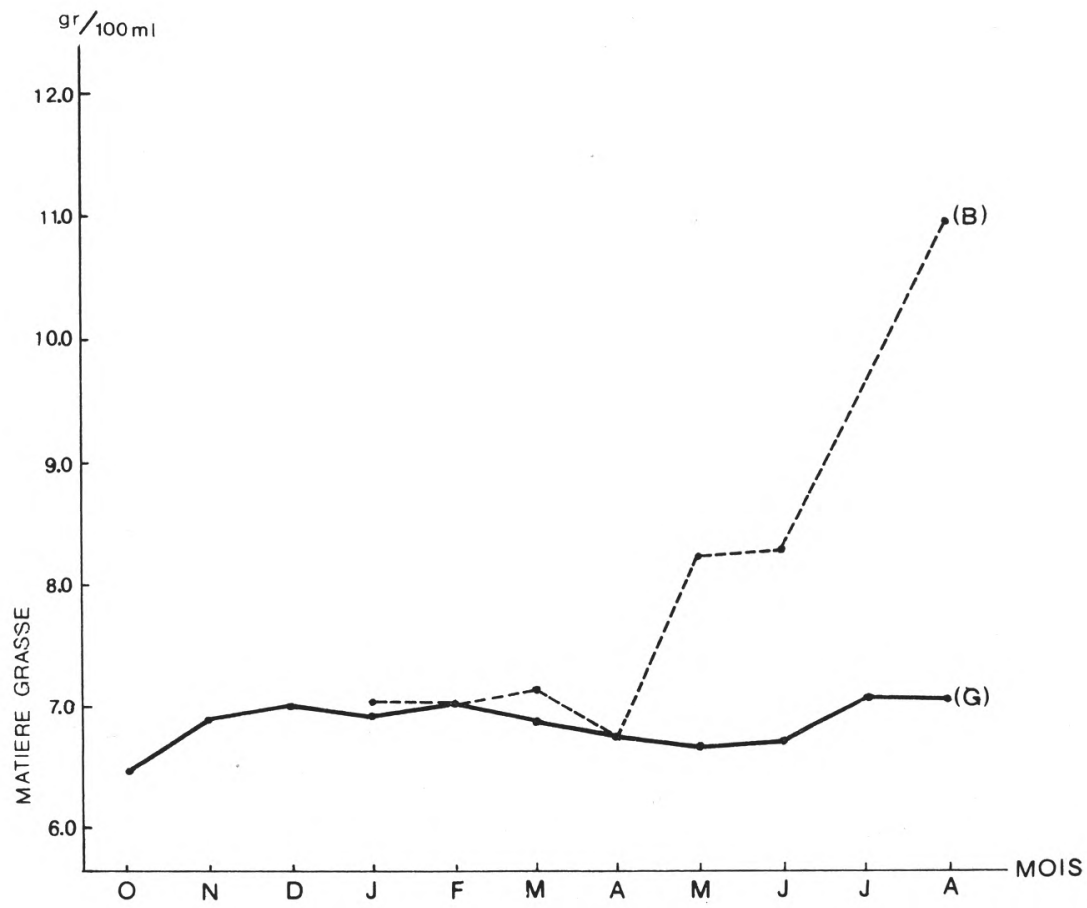

fig. 5

Variation, durant la lactation, de la teneur en extrait sec de lait de brebis de Plovdiv (B) et Ioannina (G).

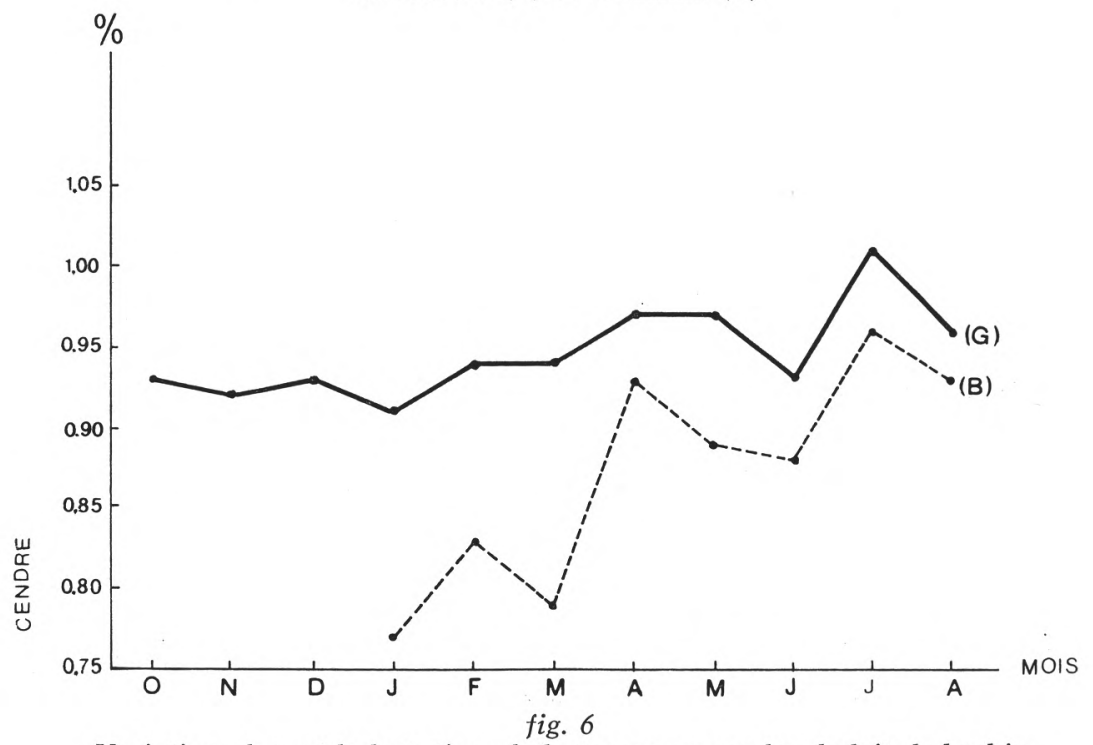

Variation, durant la lactation, de la teneur en cendre de lait de brebis de Plovdiv (B) et Ioannina (G). 
En ce qui concerne la teneur en lactose, le lait de Plovdiv contient une quantité supérieure à celle du lait de Ioannina, dont les valeurs sont les plus basses de la bibliographie. Durant la période de la lactation, la teneur en lactose est stable (fig. 4).

La teneur en extrait sec (fig. 5), pour le lait grec, est basse pendant le premier mois, stable les quatre mois qui suivent et ensuite augmente jusqu'à la fin de la lactation. Pour le lait de Plovdiv, la teneur est stable au début, s'abaisse le mois d'avril et ensuite augmente jusqu'à la fin. L'extrait sec dégraissé se montre plus stable.

Finalement, la teneur en cendres est supérieure pour le lait de Ioannina. Dans les deux cas nous avons les valeurs les plus élevées au début de la lactation (fig. 6).

Parmi les constituants des cendres nous avons déterminé le calcium, qui se présente avec des valeurs supérieures pour le lait bulgare, mais avec une plus grande variation pour le lait grec. Dans les deux cas, le lait est plus riche en calcium au début de la lactation qu'à la fin.

En dehors de la teneur en certains constituants, nous avons examiné aussi certaines caractéristiques physicochimiques comme le $\mathrm{pH}$, l'acidité et la densité, pour lesquelles nous n'avons pas constaté de différences statistiquement importantes pour le lait de deux régions.

Durant la lactation la densité et l'acidité du lait bulgare augmentent, tandis que pour le lait grec les valeurs diminuent.

\section{Rés u mé}

La composition du lait de brebis des régions Plovdiv en Bulgarie et de Ioannina en Grèce a été examinée durant les lactations des années 1979 et 1980 .

La composition moyenne de 63 échantillons de lait grec et 48 de lait bulgare, est respectivement :

Matière grasse $6,88-8,10$ p. 100 , protéines totales $5,74-5,83$ p. 100 , protéines solubles $1,44-1,33$ p. 100 , caséine $4,32-4,51$ p. 100 , lactose 4,59 4,72 p. 100 , cendres $0,95-0,87$ p. 100 , extrait sec $17,8-19,54$ p. 100 , extrait sec dégraissé $10,92-11,43$ p. 100 et calcium $0,186-0,194$ p. 100 .

Les caractéristiques physicochimiques sont respectivement : $\mathrm{pH}$ 6,58-6,57, acidité $0,22-0,21$ p. 100 en acide lactique et densité 1,0361,036 .

Les différences statistiquement significatives ont été déterminées, au seuil de 0,05 , pour la matière grasse, l'extrait sec dégraissé ou non et les cendres. Pour les trois premiers le lait bulgare est le plus riche, tandis que le lait grec est plus riche en cendres. 
Des variations ont été constatées durant la lactation pour les deux cas.

Les coefficients de variations pour le lait de Plovdiv sont plus importants pour tous les constituants, sauf le lactose et l'extrait sec dégraissé. Pour le lait de Ioannina ils sont plus important pour les protéines solubles et le calcium.

\section{Su $\mathrm{m}$ mary}

THE COMPOSITION OF SHEEP'S MILK PRODUCED IN THE PLOVDIV REGION IN BULGARIA

AND IN THE IOANNINA REGION IN THE NORTH WEST OF GREECE

A study was undertaken for the examination of the chemical composition and some, physicochemical properties of sheep's milk produced in the Plovdiv region of Bulgaria and the region of Ioannina in the north west of Greece, during the lactation periods 1979 and 1980.

The total number of samples examined was 63 Greek and 48 Bulgarian. The relewant conclusions may be summarized as follows:

1) The mean composition of the Greek and the Bulgarian sheep's milk are respectively the following per cent:

Fat 6.88-8.10, Total Protein 5.74-5.83, Water Soluble Protein 1.44-1.33, Casein 4.32-4.51, Lactose 4.59-4.72, Ash 0.95-0.87, Dry Matter 17.8019.54, S.N.F. 10.92-11.43, Calcium 0.186-0.194, Physicochemical properties: $\mathrm{pH}$ 6.58-6.57, Acidity 0.22-0.21 and Specific Gravity 1.036-1.036.

2) The chemical composition of sheep's milk of both regions varies during the lactation period.

The variability coefficients of different constituents during the lactation period are high in the bulgarian milk except for lactose and solids not fat while in the greek one were generally low, except for the weter soluble proteins and the calcium content.

3) Statistically significant differences (P-O.05) were found in the fat, total solids, the solids not fat and the ash content.

\section{Bibliographie}

[1] Anifantakis (E.), Veinoglou (B.) et Ramou (A.). - Contribution à l'étude de la composition du lait de brebis (sous presse).

[2] Balatoni (M.) (1964). - Studies of the composition and properties of ewes' milk. Doct. thesis. Dairy Sci. Abs., 26, 3039.

[3] CeRnev (P.) (1968). - Composition of ewe's milk and regularities in its. Changes depending on milking time and lactation period. Dairy Sci. Abs., $28,2948$. 
[4] GoRanov (V.) (1964). - Composition and properties of milk of fine wooled sheep recently developed for Dobruja. Dairy Sci. Abs., 26, 2370.

[5] KuBis (J.) (1963). - Study of the composition of ewes'milk during lactation. Dairy Sci. Abs., 25, 3592.

[6] LaurinciK (J.) and Mikus (M.) (1965). - Evaluation of the results obtained to date in experiments with Valachian sheep in Slovakia. Pol'nohospodarstvo, 11 (5), 409-422.

[7] Rusev (V.), Georgiev (J.) and Tanev (D.) (1965). - Study of the amount and composition of milk from different fractions of control milking of sheep. Dairy Sci. Abs., vol. 27.

[8] Veinoglou (B.), Kalatzopoulos (G.), Stamelos (N.) et Anifantakis (E.) (1968). Contribution à l'étude de la composition du lait de brebis de la race Chios. Bulletin de la Banque Agricole, 162, 1-9. 